\title{
Significant Incidence of Extra-Articular Tibia Vara Affects Radiological Outcome of Total Knee Arthroplasty
}

\author{
Balaji Saibaba, MS ${ }^{1}$, Mandeep S. Dhillon, $\mathrm{MS}^{1}$, Devendra K. Chouhan, $\mathrm{MS}^{1}$, Rajendra K. Kanojia, $\mathrm{MS}^{1}$, Mahesh \\ Prakash, $\mathrm{MD}^{2}$, and Vikas Bachhal, $\mathrm{MS}^{1}$ \\ ${ }^{1}$ Departments of Orthopaedics and ${ }^{2}$ Radiology, Post Graduate Institute of Medical Education and Research, Chandigarh, India
}

\begin{abstract}
Purpose: To identify and quantify the presence of extra-articular tibia vara that might influence the mechanical axis alignment after total knee arthroplasty (TKA).

Materials and Methods: A total of 48 TKAs in 30 osteoarthritic Indian patients were prospectively evaluated. The hip-knee-ankle angle (HKA), joint line convergence angle, and varus angulation at the femur and tibia were measured from the preoperative and postoperative standing hip-to-ankle radiographs. Four different methods were used to measure the varus angulation at the tibia: metaphyseo-diaphyseal angle (MDA), the angle between the anatomical axis and mechanical axis of the tibia, the angle between the proximal third and distal third of tibia and the angle between the proximal half and distal half of tibia.

Results: Extra-articular tibia vara quantified using MDA had the most positive correlation with HKA. Receiver operating characteristic plotting showed that MDA of $>4^{\circ}$ predicts abnormal postoperative HKA. Twenty-eight out of 48 knees had MDA of $>4^{\circ}$, and $78.6 \%$ of these had postoperative HKA under-correction and $21.4 \%$ had less than ideal tibial component position.

Conclusions: A significant inherent extra-articular varus angulation best measured using MDA exists in the proximal tibia in osteoarthritic Indian patients undergoing TKA. MDA of $>4^{\circ}$ is associated with abnormal postoperative HKA. Computer navigation may be useful for achieving ideal correction in such cases.
\end{abstract}

Keywords: Knee, Osteoarthritis, Arthroplasty, Tibia vara, Malalignment, Indian

\section{Introduction}

Survivorship of total knee arthroplasty (TKA) is dependent upon the accurate restoration of the coronal alignment of the weight-bearing axis of the lower limb and ideal placement of the femoral and tibial components perpendicular to their respective mechanical axes ${ }^{1)}$. Failure to correct the varus malalignment of

Received November 18, 2014; Revised (1st) March 13, 2015;

(2nd) May 7, 2015; (3th) June 4, 2015; (4th) July 10, 2015;

Accepted July 23, 2015

Correspondence to: Mandeep S. Dhillon, MS

Department of Orthopaedics, Post Graduate Institute of Medical

Education and Research, Sector 12, Near Punjab University, Chandigarh 160012, India

Tel: +91-172-2694774, Fax: +91-981-595-1090

E-mail: drdhillon@gmail.com

This is an Open Access article distributed under the terms of the Creative Commons Attribution Non-Commercial License (http://creativecommons.org/licenses/by-nc/4.0/) which permits unrestricted non-commercial use, distribution, and reproduction in any medium, provided the original work is properly cited. the mechanical axis of the lower limb potentially curtails the longevity of $\mathrm{TKA}^{2}$. In varus arthritic knees, the varus angulation is generally attributed to medial condylar wear, tightness of medial ligaments and laxity of lateral ligaments, which are attempted to be corrected during TKA. Apart from these explicit articular factors, the covert extra-articular (femoral and/or tibial) sources of varus malalignment of the lower limb are frequently overlooked; this might predispose to errors in bone resection and subsequent prosthesis implantation $^{3)}$. It was our perception that an inherent varus deformity in the tibia at the proximal metaphyseodiaphyseal junction, if present, may add onto the overall varus angulation at the knee. We set out to identify and quantify the presence of extra-articular varus angulation at the tibia that might influence the postoperative mechanical axis alignment of osteoarthritic patients undergoing TKA. 


\section{Materials and Methods}

A prospective study was conducted on 48 consecutive TKAs (18 bilateral and 12 unilateral TKAs) in 30 Indian patients (11 males and 19 females) with primary knee osteoarthritis. Patients with a flexion deformity of $>15^{\circ}$, mediolateral subluxation of $>3$ $\mathrm{mm}$ and those with features of inflammatory arthritis, significant spine or hip pathology, previous history of significant knee trauma or lower limb fracture or surgery on the affected lower limb were excluded from the study. In addition to the conventional anteroposterior and lateral radiographs of the knee joint, weight- bearing full-length hip-to-ankle anteroposterior "stitch radiographs" were obtained in all patients preoperatively and 6 weeks postoperatively. All the radiographs were taken with the limbs in neutral rotation and both patellae facing forward.

\section{Preoperative Radiographic Measurements}

Four parameters were measured on the preoperative radiographs: hip-knee-ankle angle (HKA), extra-articular varus angulation at the femur, joint line convergence angle (JLCA) (Fig. 1), and extra-articular varus angulation at the tibia (Fig. 2).

1) HKA was measured as the angle created between the me-
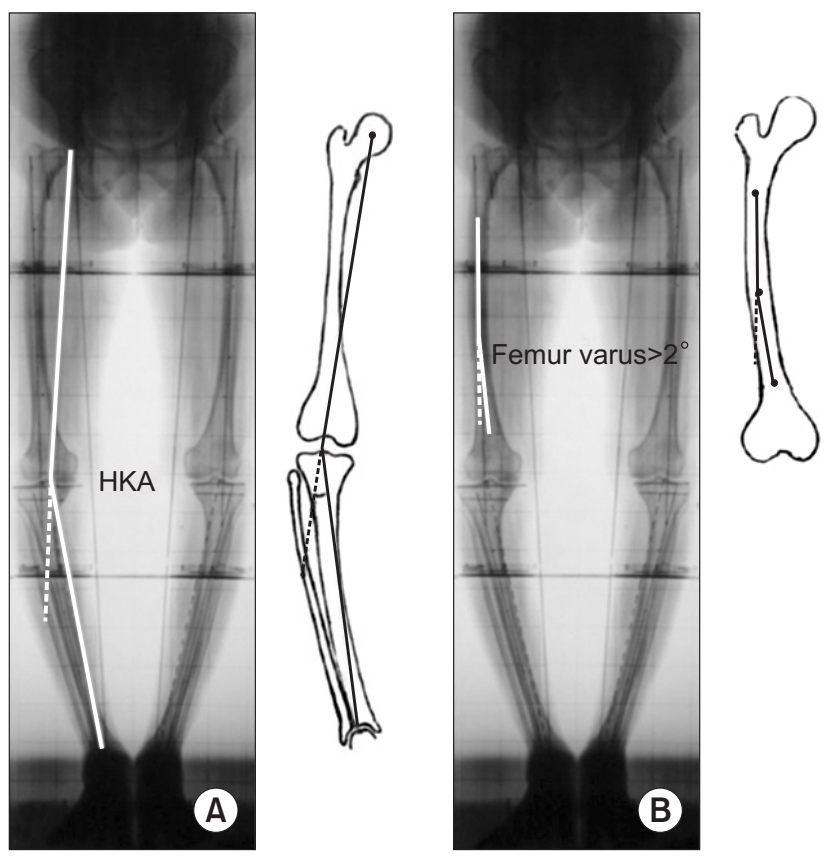

A

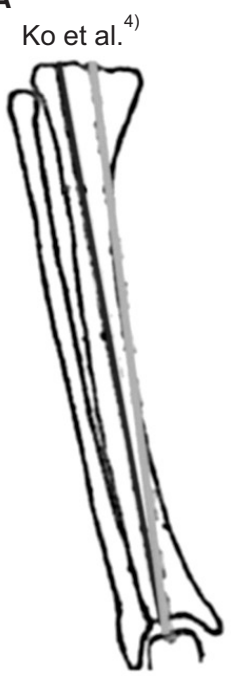

Method 1
B

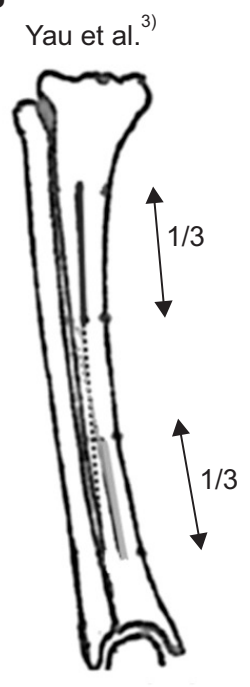

Method 2
C

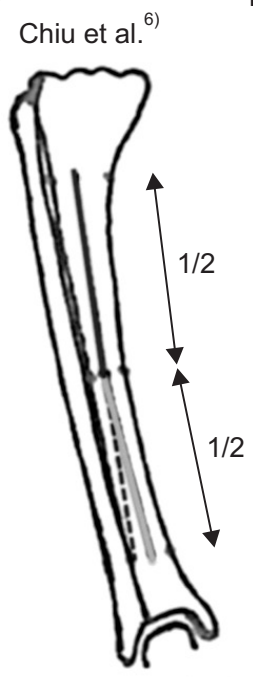

Method 3

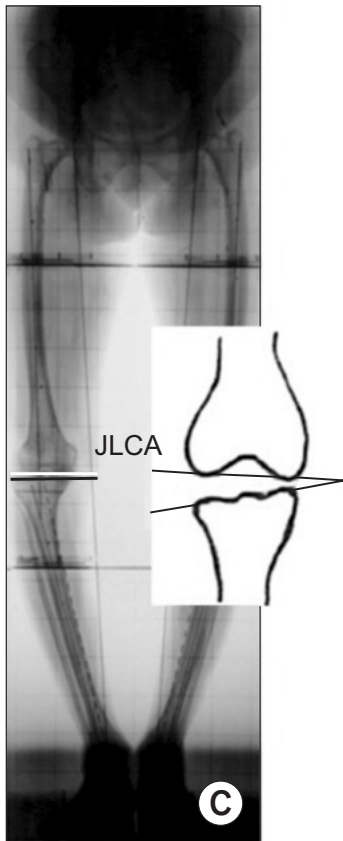

D

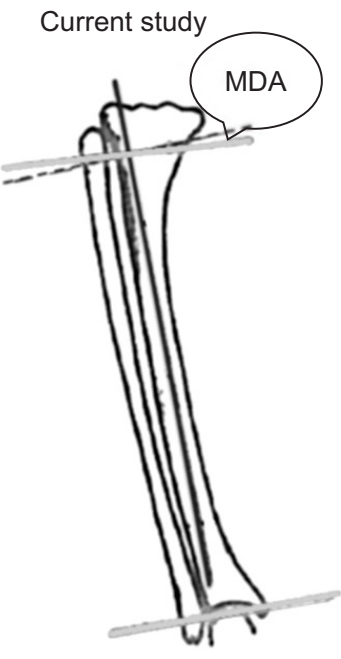

Method 4
Fig. 1. Preoperative radiographic measurements in the right limb. (A) Hip-kneeankle angle (HKA). (B) Extra-articular varus angulation at the femur. (C) Joint line convergence angle (JLCA).

Fig. 2. Four different methods to measure extra-articular tibia vara. (A) The angle between the tibial anatomical axis (black) and mechanical axis (grey). (B) The angle formed between the mid-medullary lines of the proximal and distal thirds of the tibial diaphysis. (C) The angle formed between the mid-medullary lines of the proximal and distal halves of the tibial diaphysis. (D) Using the metaphyseo-diaphyseal angle (MDA). A line is drawn at the level of proximal tibial metaphysis (grey) parallel to the ankle joint line and a second line (continuous black) is drawn connecting the mid medullary points of the tibial diaphysis intersecting the previous metaphysical line. MDA is the angle formed by the metaphysis line and a line perpendicular (interrupted black) to the diaphyseal line at this point of intersection. 
chanical axis of the femur (i.e., a line joining the centre of femoral head to the centre of intercondylar notch in the knee) and the mechanical axis of the tibia (i.e., a line joining the centre of the tibial spines to the centre of talar dome).

2) JLCA was measured as the angle formed between a line tangent to the distal femoral condyle and the proximal tibial plateau.

3) Varus angulation at the femur level was measured by the femoral diaphysis, dividing lesser trochanter (lower end) to supra condylar region into two halves. The angle formed between the mid-medullary lines of these two halves of the diaphysis denotes the varus angulation at the femur. Values greater than $2^{\circ}$ were taken as significant femoral varus angulation ${ }^{3)}$.

4) Varus angulation at the level of tibia was measured using four different methods (Fig. 2).

(1) In the first method, the varus angulation at the tibia was measured as the angle between the anatomical and mechanical axes of tibia ${ }^{4)}$. The tibial diaphysis was identified by constructing a square on the proximal and distal articular surfaces of tibia such that the dimensions of each side of the square equaled to the maximum width of the proximal and distal ends of the tibia, respectively. The area of tibia intervening the two squares was labeled as the tibial diaphysis/shaft ${ }^{5}$. Then, the tibial shaft was divided into three equal segments. The mid-medullary point of each segment was joined together to obtain the anatomical axis of tibia. Mechanical axis of the tibia was drawn by a line connecting the center of tibial spine and the center of talar dome.

(2) In the second method, the tibial diaphysis was divided into three equal segments ${ }^{3}$. The angle formed between the mid-medullary lines of the proximal and distal thirds of the tibial diaphysis was used to quantify tibial varus angulation.

(3) In the third method, the tibial diaphysis was divided into two halves ${ }^{6}$. The angle formed between the mid-medullary lines of the proximal and distal halves of the tibial diaphysis was taken as a measure of the tibial varus angulation.

(4) In the fourth method, metaphyseo-diaphyseal angle (MDA) was used to quantify the varus angulation at the tibia. A line was drawn at the level of proximal tibial metaphysis parallel to the ankle joint line. A second line was drawn connecting the midmedullary points of the tibial diaphysis intersecting the previous metaphysical line. The angle formed by the metaphysis line perpendicular to the diaphyseal line at this point of intersection was measured.

\section{Surgical Technique}

Standard surgical methods were used in all the patients. All the surgeries were performed by a single surgeon (Dhillon), who has more than 20 years of experience in arthroplasty. Preoperative planning using templates were done in all cases to estimate the ideal component size and placement. The Press Fit Condylar Sigma TKA system (DePuy, Warsaw, IN, USA) was the most widely used knee prosthesis (42 knees). In six knees, Genesis II PS system (Smith \& Nephew, Andover, MA, USA) was used. An extra-medullary alignment guide was used for proximal tibial resection in all cases. The tibial extra-medullary guide was positioned such that the two proximally placed short spikes of the zig were anchored lateral to the base of medial tibial spine. In cases where the zig did not have any spikes proximally (e.g., Depuy), the coronal alignment was set by centering the reference mark over the cutting zig at the junction of the medial one-third and middle one-third of the tibial tuberosity. The distal landmark was estimated by palpating the medial and lateral malleoli. The ankle clamp was centered over the midpoint of the talus, that is, 3 $\mathrm{mm}$ medial to the center of the ankle joint ${ }^{6,7)}$. The position of the femoral guide hole for the intra-medullary femoral guide utilized was just medial to the center of the trochlea, 5 to $10 \mathrm{~mm}$ anterior to the femoral insertion of the posterior cruciate ligament. The guide hole was subsequently drilled with a 9-mm initiator drill, which was followed by the insertion of a 9-mm intra-medullary guide. Standard methods were employed meticulously for bone resection, gap balancing, trial reduction, and final implantation.

\section{Postoperative Radiographic Measurements}

Three parameters were measured on the postoperative radiographs (Fig. 3): HKA, mechanical lateral distal femoral angle (mLDFA), and mechanical medial proximal tibial angle (mMPTA).

\section{1) Measurement of limb alignment}

The postoperative HKA was measured as the angle formed between the line joining the centre of femoral head to the centre of the intercondylar notch of the femoral implant and the line joining the centre of the tibial implant to the centre of talar dome. HKA values within $180^{\circ} \pm 3^{\circ}$ were taken as normal. Correspondingly, $\mathrm{HKA}<177^{\circ}$ was taken as varus alignment of the lower limb.

\section{2) Measurement of component positions}

(1) mLDFA was measured as the lateral angle formed between the mechanical axis of the femur (i.e., the line joining the centre of femoral head to the centre of the intercondylar notch of the femoral implant) and the tangent to the femoral implant.

(2) MMPTA was measured as the medial angle between the tangent to the tibial implant and the mechanical axis of tibia (i.e., a line joining the center of the tibial implant and the center of ta- 

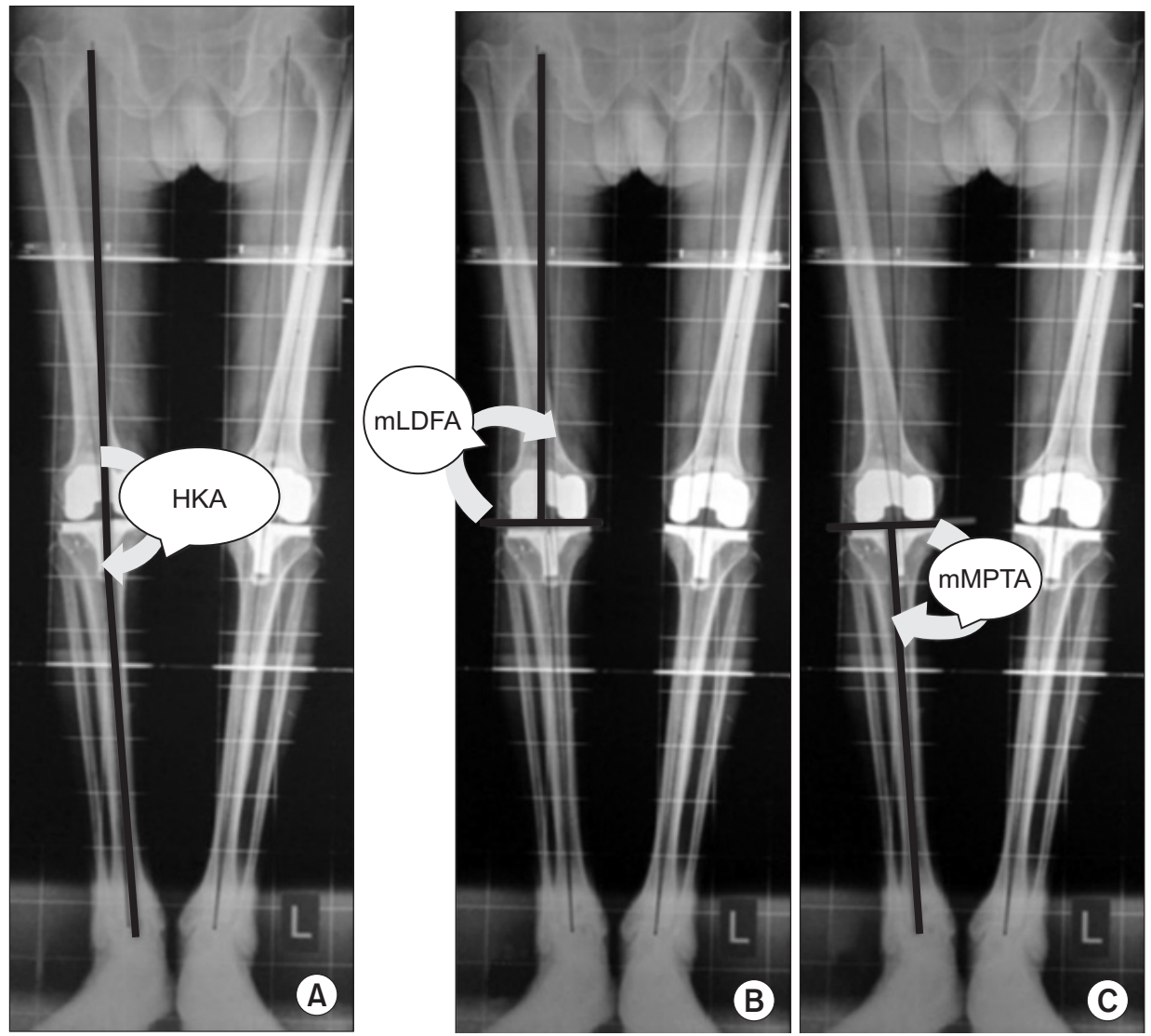

Fig. 3. Postoperative radiographic measurements in the right limb. (A) Hipknee-ankle angle (HKA). (B) Mechanical lateral distal femoral angle (mLDFA). (C) Mechanical medial proximal tibial angle (mMPTA). lar dome). Values of femoral and tibial components within $90^{\circ} \pm 3^{\circ}$ were taken as normal ${ }^{8)}$.

\section{3) Calculation of expected tibial varus angulation}

The causes of varus malalignment of the lower limb in the frontal plane include 1) femoral frontal plane deformity, 2) tibial frontal plane deformity, 3) frontal plane knee joint laxity, including subluxation and dislocation, and 4) femoral or tibial condylar deficiency ${ }^{9}$. Patients with more than $3 \mathrm{~mm}$ mediolateral subluxation were not included in the study. According to Paley ${ }^{9}$, JLCA can be used to calculate the intra-articular contribution of the varus deformity in cases where mediolateral subluxation was less than $3 \mathrm{~mm}$. Thus, varus alignment of lower limb $(\mathrm{HKA})=$ varus angulation at the femur+varus angulation at the knee (JLCA)+varus angulation at the tibia. In other words, expected varus angulation at the tibia=HKA-(femoral varus angulation+JLCA).

\section{Statistical Analysis}

The previously measured tibial varus angulation using four different methods was compared with the expected varus angulation at the tibia that was calculated by the formula given above. Spear- man's rank order correlation was used for the statistical test. A pvalue less than 0.05 was considered statistically significant. MedCalc ver. 12.5 (MedCalc Software, Ostend, Belgium) was used for statistical analysis. Receiver operating characteristic (ROC) curve was plotted with the method that was most correlated with the expected varus angulation at the tibia against the postoperative HKA. The cut-off value of the varus angulation at the tibia was specified to predict an abnormal postoperative HKA.

\section{Results}

\section{Patient Demographics}

The details of patient demographics are tabulated in Table 1. There was no statistically significant gender difference with regard to the body mass index and radiological parameters.

\section{Best Method to Quantify Tibia Vara}

The mean, standard deviation (SD), maximum, and minimum values of each radiological parameter are tabulated in Table 2.

Out of the four methods used to quantify the varus angulation at the level of tibia, MDA showed the most positive correlation with the expected tibial varus angulation (Spearman correlation 
Table 1. Patient Demographics

\begin{tabular}{lccc}
\hline \multicolumn{1}{c}{ Parameter } & Gender & Mean \pm SD (range) & p-value \\
\hline Age $(\mathrm{yr})$ & $\mathrm{M}$ & $64.1 \pm 9.0(47-78)$ & 0.330 \\
& $\mathrm{~F}$ & $61.2 \pm 6.8(49-74)$ & \\
Height $(\mathrm{cm})$ & $\mathrm{M}$ & $168.4 \pm 5.4(161-180)$ & $<0.001$ \\
& F & $160.5 \pm 4.9(150-167)$ & \\
Weight $(\mathrm{kg})$ & $\mathrm{M}$ & $78.7 \pm 4.1(72-85)$ & $<0.001$ \\
& F & $69.9 \pm 3.8(63-84)$ & \\
BMI $\left(\mathrm{kg} / \mathrm{cm}^{2}\right)$ & M & $27.4 \pm 1.1(25.2-28.8)$ & 0.230 \\
& F & $28.0 \pm 1.4(26.6-29.9)$ & \\
\hline
\end{tabular}

SD: standard deviation, BMI: body mass index.

Table 2. Summary of Preoperative (Preop) and Postoperative (Postop) Radiographic Measurements

\begin{tabular}{clc}
\hline No. & \multicolumn{1}{c}{ Parameter } & \multicolumn{1}{c}{ Value $^{\mathrm{a})}$} \\
\hline 1 & Preop HKA $\left(^{\circ}\right)$ & $-12.6 \pm 4.3(-6$ to -24$)$ \\
2 & Femoral varus $\left({ }^{\circ}\right)$ & $-0.6 \pm 1.4(0$ to -5$)$ \\
3 & Joint line convergence angle $\left(^{\circ}\right)$ & $-6.3 \pm 2.4(-3$ to -13$)$ \\
4 & Tibia vara $\left(^{\circ}\right)$ & \\
& Method 1(MDA) & $-6.1 \pm 4.1(-1$ to -19$)$ \\
& Method 2 (AA-MA) & $-1.1 \pm 1.0(0$ to -3$)$ \\
& Method 3 $(1 / 2-1 / 2)$ & 0 \\
& Method 4 $(1 / 3-1 / 3)$ & $-5.7 \pm 3.6(-15$ to 1$)$ \\
& Expected varus ${ }^{\text {b) }}$ & $-5.5 \pm 3.2(-1$ to -11$)$ \\
6 & Postop HKA $\left({ }^{\circ}\right)$ & $90.1 \pm 2.7(84$ to 98$)$ \\
7 & Postop mLDFA $\left({ }^{\circ}\right)$ & $87.7 \pm 2.5(83$ to 92$)$ \\
\hline
\end{tabular}

Values are presented as mean \pm standard deviation (range).

HKA: hip-knee-ankle angle, MDA: metaphyseo-diaphyseal angle, AA: anatomical axis, MA: mechanical axis, mLDFA: mechanical lateral distal femoral angle, mMPTA: mechanical medial proximal tibial angle.

${ }^{a)}$ Minus (-) indicates varus, plus (+) indicates valgus (in degrees)

${ }^{b}$ Expected varus in tibia=HKA-(femoral varus+joint line convergence angle)

coefficient, 0.47 ) with a p-value of 0.001 . The ROC curve showed that $\mathrm{MDA}>4^{\circ}$ predicts abnormal post-operative HKA (beyond $180^{\circ} \pm 3^{\circ}$ ) with a sensitivity of $78.1 \%$ and specificity of $62.5 \%$ and with a p-value of 0.03 .

\section{Incidence of Tibia Vara}

Preoperatively, 28 out of 48 knees (58.3\%) had MDA $>4^{\circ}$, ranging from $5^{\circ}$ to $19^{\circ}$ with a mean of $9^{\circ}$ and $\mathrm{SD}$ of $2.8^{\circ}$.

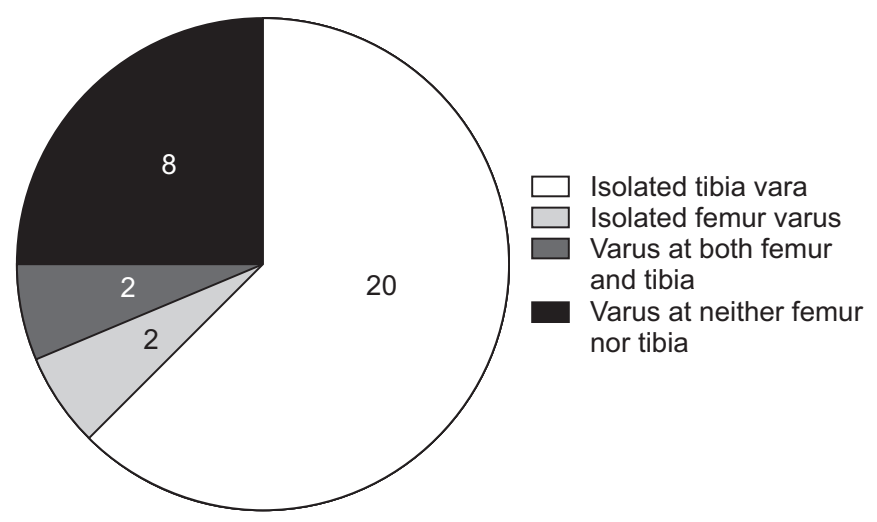

Fig. 4. Pie diagram showing the distribution of knees with hip-kneeankle angle under-correction $(\mathrm{n}=32)$.

\section{Impact of Tibia Vara on HKA Correction}

Postoperatively, 32 out of 48 knees had significant under-correction of HKA $\left(<177^{\circ}\right)$. Out of these, isolated tibial varus angulation $\left(\mathrm{MDA}>4^{\circ}\right)$ was observed in 20 knees, isolated femoral varus angulation, in two knees $\left(>2^{\circ}\right)$, and varus angulation at both tibia and femur, in two knees (Fig. 4). Eight knees had an abnormal postoperative HKA $\left(<177^{\circ}\right)$ without varus angulation at the tibia $\left(\mathrm{MDA} \leq 4^{\circ}\right)$ and femur $\left(<2^{\circ}\right)$. Six knees had an abnormal MDA $\left(>4^{\circ}\right)$ but a normal postoperative HKA (i.e., within $180^{\circ} \pm 3^{\circ}$ ).

Of those with extra-articular tibia vara, $78.6 \%$ had postoperative HKA under-correction. In all those 22 patients who had a varus angulation at the tibia $\left(\mathrm{MDA}>4^{\circ}\right)$ along with an abnormal postoperative HKA $\left(<177^{\circ}\right)$, the degree of under-correction of HKA had a statistically significant correlation with the varus angulation at the tibia, i.e., MDA (Pearson correlation coefficient [PCC], -0.47; p-value, 0.02). On the contrary, in all those eight knees that had no varus angulation at either femur or tibia but an abnormal postoperative HKA, the degree of under-correction of HKA did not have a statistically significant correlation with MDA (PCC, -0.11 ; p-value, 0.79).

\section{Influence of Tibia Vara on Tibial Component Placement}

Less than ideal component position (either mMPTA and/or mLDFA beyond $90^{\circ} \pm 3^{\circ}$ ) was observed in 24 out of 48 knees: 14 knees with isolated tibial component malposition, 6 knees with isolated femoral component malposition, and 4 knees with both components in malposition. Excluding all those cases with femoral component malposition, the incidence of tibial component position being less than ideal was $36.8 \%$ ( 14 out of 38 knees). Out of these, 6 knees $(42.6 \%)$ had been documented as tibia vara. In other words, 6 out of 28 knees (21.4\%) with abnormal MDA of $>4^{\circ}$ had subsequent positioning of the tibial component in a less 
than ideal position.

\section{Discussion}

Varus malalignment in osteoarthritic knee is multifactorial in origin. The articular sources of varus malalignment (excessive medial articular wear, lateral subluxation of tibia, tight medial collateral ligament (MCL) and/or lax lateral collateral ligament) are usually evident intraoperatively and are rectified during TKA. On the other hand, other possible extra-articular sources of varus malalignment, such as femoral and tibial varus angulation, are usually concealed clinically and are easily overlooked. At the initiation of this study, it was our belief that an unrecognized, yet significant, inherent extra-articular varus angulation could exist at the proximal metaphyseo-diaphyseal junction of the tibia in patients having varus arthritic knees. This varus angulation at the tibia might be responsible for inadequate restoration of mechanical axis of the lower limb after TKA and thereby impairs the longevity of TKA. On reviewing the literature, we could not find any evidence documenting the incidence of extra-articular varus angulation in the proximal metaphyseo-diaphyseal junction of the tibia in Indian patients, and its effect, if any, on TKA.

Careful preoperative radiological evaluation is pivotal in the identification of the varus angulation at the proximal tibia. Coronal bowing of tibia is best identified by a stitched X-ray, including the hip-to-ankle in a standing position ${ }^{10}$. In addition to the identification of tibial bowing, these weight- bearing full-length radiographs are instrumental in the assessment of the mechanical axis of the lower limb.

Till date, four methods have been described for the quantification of tibial bowing or, in other words, varus angulation at the tibia. Ko et al. ${ }^{4)}$ quantified tibial varus deformity by measuring the angle formed between the mechanical and the anatomical axes of the tibia. Tibia vara was said to be present if this angle was greater than $2^{\circ}$. Yau et al. ${ }^{3)}$ considered tibial bowing to be present if there was more than $2^{\circ}$ angulation between the proximal and distal third of the tibial diaphysis. Chiu et al. ${ }^{6}$ considered tibial bowing to be present if the angle formed between the anatomical axes of the proximal half and the distal half of the tibial diaphysis was more than $2^{\circ}$. Levine and Drennan ${ }^{11)}$ considered tibia vara to be present if the MDA was greater than $11^{\circ}$ in the paediatric population.

We used a similar methodology, with a slight modification, to quantify the varus angulation at the tibia in the osteoarthritic population. Levine and Drennan ${ }^{11)}$ used a metaphyseal line which passed through the most distal ossified peak of the medial and lateral beaks of the tibial metaphysis. Since this is not possible in adult population, we drew a line through the proximal tibial metaphysis which was parallel to the ankle joint line. We did not take the knee joint line (i.e., a tangent through the deepest points of medial and lateral tibial plateaus) as the reference because of the asymmetric nature of the articular wear which is commonly seen in varus osteoarthritic knees.

Since we presumed that most of the extra-articular varus deformity existed in the metaphysio-diaphyseal junction, we modified the method proposed by Levine and Drennan ${ }^{11)}$ that measures the MDA. The existing methods are known to measure deformity far distally which could be erroneous; however, there is no evidence to support our modification of Levine and Drennan's method. It is proposed by us to accurately measure this contentious issue. Our method of quantifying the varus angulation at the tibia using MDA had the most positive correlation with the expected tibial varus angulation. This method is simple and easily reproducible. Further, our study also determines the cutoff value for preoperative MDA, which helps in predicting an abnormal postoperative HKA (i.e., beyond $180^{\circ} \pm 3^{\circ}$ ). MDA value greater than $4^{\circ}$ is associated with a postoperative varus HKA with a sensitivity of $78.1 \%$ and specificity of $62.5 \%$.

Twenty-two out of 28 knees (78.6\%) that had preoperative MDA of $>4^{\circ}$ had varus under- correction of HKA after TKA using standard extra-medullary alignment jigs. Similarly, six out of 28 knees (21.4\%) that had preoperative MDA of $>4^{\circ}$ had tibial component malposition after TKA. Surgeons need to keep this in mind while planning the tibial cut during TKA. On retrospective analysis, we think that by increasing the magnitude of tibial resection laterally, we could have achieved better restoration of the mechanical axis of the lower limb in this subgroup. On the other hand, the effect of larger lateral cuts on the tibial component alignment is questionable. If the extra-articular tibial varus can be accurately identified preoperatively, then the following modifications can be made in the conventional surgical procedure of TKA while using extra-medullary guide system in those with tibia vara. According to Wang and Wang ${ }^{12)}$, intra-articular bone resection and soft tissue balancing during TKA can be successful in patients with arthritis of the knee associated with an extraarticular tibial varus deformity of up to 20 degrees. The bone resection from the lateral tibial condyle should be limited to $1 \mathrm{~cm}$ and the remaining defect is to be filled with bone graft. Soft tissue balancing can be achieved by sequential release of the tight medial soft tissue structures as advised by Mullaji et al. ${ }^{13)}$. The deep MCL, posteromedial capsule, and semimembranosus are subperiosteally elevated as a part of the initial exposure during TKA. 
Subsequently after tibial resection, reduction osteotomy of the proximal tibia, sub-periosteal elevation of the superficial MCL and partial or complete release of the pes anserinus insertion can be sequentially performed to relieve the residual medial tightness. Various studies have shown that tibial resection using intramedullary alignment systems produce superior results to extramedullary systems during $\mathrm{TKA}^{8,14-17)}$. The intra-medullary alignment rod has to be inserted parallel to the mechanical axis of tibia for performing an optimal tibial resection ${ }^{18)}$. This, in turn, depends upon the degree of parallelism between the anatomic and mechanical axes of tibia ${ }^{3)}$. Studies have also shown that significant bowing in the tibia precludes the use of intra-medullary alignment guides for proximal tibial resection during TKA ${ }^{3,14,19,20)}$. Yau et al. $^{3)}$ and Ko et al. ${ }^{4)}$ observed that the use of tibial intramedullary alignment guide for proximal tibial resection resulted in unacceptable tibial cut in the presence of tibial bowing because the deformity made the passage of the intra-medullary rod parallel to the tibial mechanical axis impossible. On the other hand, extra-medullary alignment jigs have been shown to have a wide range of accuracy of tibial component placement, ranging from $65 \%$ to $88 \%^{18,21)}$. In our study, $36.8 \%$ of the knees had less than ideal tibial component position; extra-medullary alignment guide was successful in achieving optimal tibial resection and component placement in $63.2 \%$ of the knees. Our result closely resembles that of Reed et al. ${ }^{18)}$ in which optimal tibial alignment was achieved in $65 \%$ of the cases using extra-medullary alignment guides. In modern orthopaedics, with the advent of computer-assisted navigation systems, the accuracy of tibial resection and tibial component placement can reach up to $100 \%{ }^{22)}$. These navigated surgeries not only help in improving the precision of implant positioning, but also, in facilitating accurate restoration of the mechanical axis of the lower limb ${ }^{23-26)}$. Patients with osteoarthritis of the knee with an inherent varus deformity in the tibia, in whom neither intra-medullary guides can be utilized nor does extra-medullary guides give consistent results, might be the ideal candidates for computer-assisted arthroplasty in the future.

\section{Conclusions}

Inherent extra-articular varus deformity exists in the proximal tibia in a significant percentage of Indian patients with osteoarthritis undergoing TKA. Existing methods underestimate the deformity and we propose MDA as a more reliable and precise method to measure tibia vara. An MDA greater than $4^{\circ}$ was found to have a statistically significant influence on postoperative HKA. Hence, screening for extra-articular tibia vara must be included in the preoperative planning as a routine. Utmost care is to be taken while operating such patients; computer-navigated TKA can be suggested, if the degree of tibia vara is considered significant.

\section{Conflict of Interest}

No potential conflict of interest relevant to this article was reported.

\section{References}

1. Insall JN, Binazzi R, Soudry M, Mestriner LA. Total knee arthroplasty. Clin Orthop Relat Res. 1985;(192):13-22.

2. Denham RA, Bishop RE. Mechanics of the knee and problems in reconstructive surgery. J Bone Joint Surg Br. 1978; 60:345-52.

3. Yau WP, Chiu KY, Tang WM, Ng TP. Coronal bowing of the femur and tibia in Chinese: its incidence and effects on total knee arthroplasty planning. J Orthop Surg (Hong Kong). 2007;15:32-6.

4. Ko PS, Tio MK, Ban CM, Mak YK, Ip FK, Lam JJ. Radiologic analysis of the tibial intramedullary canal in Chinese varus knees: implications in total knee arthroplasty. J Arthroplasty. 2001;16:212-5.

5. Muller ME. Nazarian S, Koch P, Schatzker J. The comprehensive classification of fractures of long bones. Berlin: Springer-Verlag; 1990. p144-5.

6. Chiu KY, Yau WP, Ng TP, Tang WM. The accuracy of extramedullary guides for tibial component placement in total knee arthroplasty. Int Orthop. 2008;32:467-71.

7. Moreland JR. Mechanisms of failure in total knee arthroplasty. Clin Orthop Relat Res. 1988;(226):49-64.

8. Jeffery RS, Morris RW, Denham RA. Coronal alignment after total knee replacement. J Bone Joint Surg Br. 1991;73:709-14.

9. Paley D. Malalignment and malorientation in frontal plane. In: Paley D, ed. Principles of deformity correction. Berlin: Springer; 2002. p19.

10. McGrory JE, Trousdale RT, Pagnano MW, Nigbur M. Preoperative hip to ankle radiographs in total knee arthroplasty. Clin Orthop Relat Res. 2002;(404):196-202.

11. Levine AM, Drennan JC. Physiological bowing and tibia vara: the metaphyseal-diaphyseal angle in the measurement of bowleg deformities. J Bone Joint Surg Am. 1982;64:1158-63.

12. Wang JW, Wang CJ. Total knee arthroplasty for arthritis of the knee with extra-articular deformity. J Bone Joint Surg 
Am. 2002;84:1769-74.

13. Mullaji AB, Padmanabhan V, Jindal G. Total knee arthroplasty for profound varus deformity: technique and radiological results in 173 knees with varus of more than 20 degrees. J Arthroplasty. 2005;20:550-61.

14. Maestro A, Harwin SF, Sandoval MG, Vaquero DH, Murcia A. Influence of intramedullary versus extramedullary alignment guides on final total knee arthroplasty component position: a radiographic analysis. J Arthroplasty. 1998;13:552-8.

15. Teter KE, Bregman D, Colwell CW Jr. Accuracy of intramedullary versus extramedullary tibial alignment cutting systems in total knee arthroplasty. Clin Orthop Relat Res. 1995;(321):106-10.

16. Brys DA, Lombardi AV Jr, Mallory TH, Vaughn BK. A comparison of intramedullary and extramedullary alignment systems for tibial component placement in total knee arthroplasty. Clin Orthop Relat Res. 1991;(263):175-9.

17. Elloy MA, Manning MP, Johnson R. Accuracy of intramedullary alignment in total knee replacement. J Biomed Eng. 1992;14:363-70.

18. Reed MR, Bliss W, Sher JL, Emmerson KP, Jones SM, Partington PF. Extramedullary or intramedullary tibial alignment guides: a randomised, prospective trial of radiological alignment. J Bone Joint Surg Br. 2002;84:858-60.

19. Bono JV, Roger DJ, Laskin RS, Peterson MG, Paulsen CA. Tibial intramedullary alignment in total knee arthroplasty. Am J Knee Surg. 1995;8:7-11.
20. Yang SH, Liu TK. Intramedullary versus extramedullary tibial alignment guides in total knee arthroplasty. J Formos Med Assoc. 1998;97:564-8.

21. Dennis DA, Channer M, Susman MH, Stringer EA. Intramedullary versus extramedullary tibial alignment systems in total knee arthroplasty. J Arthroplasty. 1993;8:43-7.

22. Wong T, Chen SH, Ko JY, Wang FS, Chou WY, Chen CM. Computer-assisted navigation system helps experienced surgeon improve outcome in total knee arthroplasty. Life Sci J. 2012;9:185-90.

23. Haaker RG, Stockheim M, Kamp M, Proff G, Breitenfelder J, Ottersbach A. Computer-assisted navigation increases precision of component placement in total knee arthroplasty. Clin Orthop Relat Res. 2005;(433):152-9.

24. Tyagi V, Kim TH, Hwang JH, Oh KJ. Imageless navigation assisted total knee arthroplasty with comprehensive gap balancing in medial osteoarthritic varus knees with anatomic variations. Comput Aided Surg. 2010;15:90-7.

25. Huang TW, Hsu WH, Peng KT, Hsu RW, Weng YJ, Shen WJ. Total knee arthroplasty with use of computer-assisted navigation compared with conventional guiding systems in the same patient: radiographic results in Asian patients. J Bone Joint Surg Am. 2011;93:1197-202.

26. Rhee SJ, Seo CH, Suh JT. Navigation-assisted total knee arthroplasty for patients with extra-articular deformity. Knee Surg Relat Res. 2013;25:194-201. 